\title{
以新型 $\left[\mathrm{Mo}_{8} \mathrm{Na}_{2} \mathrm{O}_{28}\right]^{6-}$ 多阴离子为建筑单元的 2D $\rightarrow 3 \mathrm{D}$ 互锁配位聚合物的组装、结构及性质
}

王秀丽”，李锦，田爱香，刘国成，林宏艳

渤海大学化学化工学院, 锦州 121000

*通讯作者, E-mail: wangxiuli@ @bhu.edu.cn

收稿日期: 2010-11-03; 接受日期: 2010-12-23

doi: 10.1360/032010-810

摘要以 $\mathrm{NiCl}_{2} 、\left(\mathrm{NH}_{4}\right)_{6} \mathrm{Mo}_{7} \mathrm{O}_{24} 、 \mathrm{NaOH}$ 及柔性配体 1,3-二(1,2,4-三氮唑-1-)丙烷(Btp) 为 原料, 采用水热技术合成了一个结构新颖的基于多金属氧酸盐(多酸) $\left[\mathrm{Mo}_{8} \mathrm{Na}_{2} \mathrm{O}_{28}\right]^{6}$-的二维 有机-无机杂化配位聚合物 $\left[\mathrm{Ni}(\mathrm{Btp})_{2}\left(\mathrm{H}_{2} \mathrm{O}\right)\right]\left[\mathrm{Mo}_{8} \mathrm{Na}_{2} \mathrm{O}_{26}\left(\mu_{2}-\mathrm{OH}\right)_{2}\right]_{0.5} \cdot \mathrm{H}_{2} \mathrm{O}(\mathbf{1})$. 通过元素分 析、IR、TG 和 X-射线单晶衍射等对化合物 $\mathbf{1}$ 进行了表征. X-射线单晶结构分析表明, 该 化合物属于单斜晶系, $P 2_{1} / c$ 空间群, 晶胞参数 $a=11.6871(8) \AA, b=21.7506(15) \AA, c=$ 14.9208(8) $\AA, Z=4, R_{1}=0.0487, w R_{2}=0.1409$. 化合物 1 中含有一种新型的多酸阴离子簇 $\left[\mathrm{Mo}_{8} \mathrm{Na}_{2} \mathrm{O}_{28}\right]^{6-}$, 可以看作是由 $\beta-\left[\mathrm{Mo}_{8} \mathrm{O}_{26}\right]^{4-}$ 转化成的具有 $\left[\mathrm{V}_{10} \mathrm{O}_{28}\right]^{6-}$ 结构特征的新型多酸. 该多酸被镍和 Btp 形成的二维 (2D) 层夹在中间, 形成一种夹心型的 $2 \mathrm{D}$ 柱状层结构. 二 维柱状层之间相互穿插形成了一个二维 $\rightarrow$ 三维 $(2 \mathrm{D} \rightarrow 3 \mathrm{D})$ 的互锁结构, 柱状层之间的氢键 作用又进一步稳固了整个框架. 此外, 该化合物具有较好的电催化和光催化性能.

关键词

多金属氧酸盐

$2 \mathrm{D}$ 互锁结构

配位聚合物

电化学和荧光性质

光催化活性

\section{1 引言}

多金属氧酸盐 (多酸)作为一大类多核簇合物, 具有独特的分子结构及物理、化学性质, 它们在催 化、吸附、光学、磁性等功能材料方面具有潜在的应 用前景, 受到人们广泛关注 ${ }^{[1 \sim 6]}$. 近年来, 人们已设 计合成了大量的以多酸为基本建筑单元的功能化材 料 ${ }^{[7 \sim 11]}$. 同多钿酸盐 $\left[\mathrm{Mo}_{8} \mathrm{O}_{26}\right]^{4-}$ 作为多酸家族中的重 要一员, 因具有可变的结构模式, 在构筑多酸基杂化 材料方面引起人们很大的兴趣. 迄今为止, 已被报道 过的 $\left[\mathrm{Mo}_{8} \mathrm{O}_{26}\right]^{4}$ 的异构体形式包括 $\alpha, \beta, \gamma, \delta, \varepsilon, \zeta, \eta$ 和 $\theta$ 型共 8 种 ${ }^{[12 \sim 16]}$.

$\left[\mathrm{Mo}_{8} \mathrm{O}_{26}\right]^{4-}$ 阴离子作为构筑单元可以被过渡金属 离子 $\mathrm{Cu}^{\mathrm{II}}, \mathrm{Co}^{\mathrm{II}}, \mathrm{Ni}^{\mathrm{II}}, \mathrm{Ag}^{\mathrm{I}}$ 等通过端氧或桥氧修饰, 并扩 展形成不同维度的网络结构 ${ }^{[12,14,17 ~ 18]}$. 碱金属 $\mathrm{Na}^{\mathrm{I}}$ 修
饰其他多酸阴离子构筑的框架结构已有报道 ${ }^{[19 \sim 21]}$, 但 $\mathrm{Na}^{\mathrm{I}}$ 修饰 $\left[\mathrm{Mo}_{8} \mathrm{O}_{26}\right]^{4-}$ 阴离子形成混合多金属氧簇, 并作为构筑单元进一步和过渡金属-有机配体链接构 成的高维框架结构目前还未见报道。

大量实验结果表明，水热合成技术与多酸及金 属-有机分子的结合是合成结构新颖的多酸基杂化物 的有效方法. 目前, 许多高维的、高连接的具有穿插 (自穿插和互穿)、多轮烷、缠绕等新颖结构特点的多 酸基金属-有机框架已被报道 ${ }^{[22 ~ 24]}$. 虽然三维无限延 伸的金属有机框架和索烃已有研究, 然而与索烃具 有相似特点的互锁结构的多酸基金属-有机杂化物的 报道却非常有限 ${ }^{[25,26]}$.

本文采用水热技术合成了一个结构新奇的

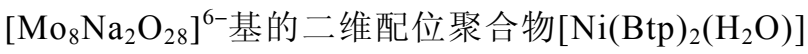


$\left[\mathrm{Mo}_{8} \mathrm{Na}_{2} \mathrm{O}_{26}\left(\mu_{2}-\mathrm{OH}\right)_{2}\right]_{0.5} \cdot \mathrm{H}_{2} \mathrm{O}(\mathbf{1})(\mathrm{Btp}=1,3-$ 二 $(1,2,4-$ 三 氮唑-1-)丙烷), 该结构包括一个由金属-有机单元组 成的二维格子, 通过一种新型多酸 $\left[\mathrm{Mo}_{8} \mathrm{Na}_{2} \mathrm{O}_{28}\right]^{6-}$ 阴 离子的链接形成一个夹心型的二维柱状层, 柱状层 之间以一种交错的方式互锁, 最后构成一个有趣的 $2 \mathrm{D} \rightarrow 3 \mathrm{D}$ 的互锁结构. 本文研究了标题化合物的电催 化和光催化性能.

\section{2 实验部分}

\section{1 试剂与仪器}

所用试剂均为分析纯, 未经进一步纯化直接使 用. 配体 Btp 根据文献合成 ${ }^{[27]}$. C, H, N 元素分析使用 Perkin Elmer 240C 元素分析仪(美国)测定, 热重分析 使用 Pyris 热重分析仪(美国)测定, IR 光谱用 Magna FT-IR560 型红外光谱仪 ( KBr 压片)测定, 电化学性质 使用 CHI 440 型电化学石英晶体微天平(上海)测定, 荧光光谱使用 HITACHI F-4500 (日本)荧光光谱仪测 定, 光催化性能使用 SP-1900UV 型双光束紫外可见 分光光度计(上海)测定, 单晶结构使用 Bruker Smart 1000 CCD 型 X-射线单晶衍射仪(德国)测定.

\section{$2.2\left[\mathrm{Ni}(\mathrm{Btp})_{2}\left(\mathrm{H}_{2} \mathrm{O}\right)\right]\left[\mathrm{Mo}_{8} \mathrm{Na}_{2} \mathrm{O}_{26}\left(\mu_{2}-\mathrm{OH}\right)_{2}\right]_{0.5} \cdot \mathrm{H}_{2} \mathrm{O}$} 的合成(1)

将 $\mathrm{NiCl}_{2} \cdot 6 \mathrm{H}_{2} \mathrm{O}(0.12 \mathrm{~g}, 0.5 \mathrm{mmol})$, Btp $(0.043 \mathrm{~g}$, $0.2 \mathrm{mmol}),\left(\mathrm{NH}_{4}\right)_{6} \mathrm{Mo}_{7} \mathrm{O}_{24} \cdot 4 \mathrm{H}_{2} \mathrm{O}(0.124 \mathrm{~g}, 0.1 \mathrm{mmol})$, $\mathrm{H}_{2} \mathrm{O}(10 \mathrm{~mL})$ 和 $\mathrm{NaOH}(0.3 \mathrm{~mL}, 1.0 \mathrm{~mol} / \mathrm{L})$ 在烧杯中混 合, 置于搅拌器上搅拌 $60 \mathrm{~min}$, 用 $1.0 \mathrm{~mol} / \mathrm{L}$ 的盐酸 调节溶液 $\mathrm{pH}$ 值为 1.3 , 然后将此混合物置于具有聚四 氟乙烯内祄的不锈钢反应釜中, $160^{\circ} \mathrm{C}$ 下晶化 $3 \mathrm{~d}$, 自 然冷却至室温, 得到蓝色块状晶体. 晶体过滤并用蒸 馏水清洗, 在室温下放置干燥, 产率约为 $20 \%$ (以 $\mathrm{Ni}$ 计算). 元素分析: 按分子式 $\mathrm{C}_{14} \mathrm{H}_{25} \mathrm{Mo}_{4} \mathrm{~N}_{12} \mathrm{NaNiO}_{16}$ 计 算值(\%) C 15.53, H 2.33, N 15.53; 实验值(\%) C 15.61, H 2.18, N 15.33. 红外光谱 IR $\left(\mathrm{KBr}, \mathrm{cm}^{-1}\right)$ : $3443 \mathrm{w}$, $3112 \mathrm{~m}, 1635 \mathrm{~s}, 1531 \mathrm{~s}, 1450 \mathrm{w}, 1290 \mathrm{~s}, 1382 \mathrm{w}, 1125 \mathrm{~s}$, $995 \mathrm{w}, 951 \mathrm{~s}, 912 \mathrm{~s}, 845 \mathrm{~m}, 721 \mathrm{~s}, 675 \mathrm{~m}$.

\section{3 化合物 1 本体修饰碳糊电极的制备}

将 $0.5 \mathrm{~g}$ 石墨粉与 $30 \mathrm{mg}$ 标题化合物混合后在玛 瑙研钭中研磨 $30 \mathrm{~min}$, 得到均匀的混合物. 向混合物 中加入 $0.15 \mathrm{~mL}$ 石蜡油, 搅拌均匀后将这种碳糊装入 一个内径 $3 \mathrm{~mm}$ 的玻璃管中压实, 封装长度 $0.8 \mathrm{~cm}$.
表面用称量纸擦平. 空白碳糊电极的制法与上述相 同，只是没有加入修饰剂(化合物 $\mathbf{1}$ ).

\section{4 晶体结构的测定}

选取尺寸为 $0.15 \mathrm{~mm} \times 0.20 \mathrm{~mm} \times 0.24 \mathrm{~mm}$ 的晶 体用于单晶结构解析. 用石墨单色器单色化的 $\mathrm{Mo}$ $\mathrm{K} \alpha$ 射线 $(\lambda=0.071073 \mathrm{~nm})$, 在 $1.87^{\circ} \leqslant \theta \leqslant 26.00^{\circ}$ 范围 内共收集 16591 个衍射点, 其中独立的衍射点为 6044 个 $\left(R_{\mathrm{int}}=0.0372\right)$. 数据还原在 BRUKER SAINT 程序 上进行, 部分结构的衍射数据使用 SADABS 程序进 行吸收校正. 晶体结构由直接法结合差值 Fourier 合 解 ${ }^{[28]}$. 全部非氢原子坐标及各向异性参数进行全矩 阵最小二乘法修正. $\mathrm{H}_{2} \mathrm{O}$ 上氢原子由差值 Fourier 图合 成法得到, 其他氢原子坐标为理论加氢而得. 配合物 的最终一致性因子为 $R_{1}=0.0487, w R_{2}=0.1409$. 标题 化合物的晶体学参数和主要键长见表 1 和表 2 . 晶体 的 CCDC 号为 798291 .

\section{3 结果与讨论}

\section{1 晶体结构}

$\mathrm{X}$-射线单晶结构分析表明, 化合物 $\mathbf{1}$ 是一个二维

表 1 化合物 1 的晶体学参数

\begin{tabular}{|c|c|}
\hline 分子式 & $\mathrm{C}_{14} \mathrm{H}_{25} \mathrm{Mo}_{4} \mathrm{~N}_{12} \mathrm{NaNiO}_{16}$ \\
\hline 分子量 & 1082.92 \\
\hline 晶体结构 & 单斜晶体 \\
\hline 空间点群 & $P 2_{1} / c$ \\
\hline$a(\AA)$ & $11.6871(8)$ \\
\hline$b(\AA)$ & $21.7506(15)$ \\
\hline$c(\AA)$ & $14.9208(8)$ \\
\hline$\alpha\left({ }^{\circ}\right)$ & 90 \\
\hline$\beta\left(^{\circ}\right)$ & $125.951(4)$ \\
\hline$\gamma\left({ }^{\circ}\right)$ & 90 \\
\hline$V\left(\AA^{3}\right)$ & $3070.4(3)$ \\
\hline$D / \mathrm{g} \mathrm{cm}^{-3}$ & 2.340 \\
\hline$Z$ & 4 \\
\hline$F(000)$ & 2108 \\
\hline$M\left(\mathrm{~mm}^{-1}\right)$ & 2.295 \\
\hline$T(\mathrm{~K})$ & $296(2)$ \\
\hline$\theta$ range $\left(^{\circ}\right)$ & $1.87 \sim 26.00$ \\
\hline$R_{\text {int }}\left(\right.$ on $\left.F^{2}\right)$ & 0.0372 \\
\hline$R_{1}^{a)}[I>2 \sigma(I)]$ & 0.0487 \\
\hline$w R_{2}^{b)}$ (all data) & 0.1409 \\
\hline GOF on $F^{2}$ & 1.050 \\
\hline$\Delta \rho_{\max }\left(\mathrm{e} \AA^{-3}\right)$ & 0.937 \\
\hline$\Delta \rho_{\min }\left(\mathrm{e} \AA^{-3}\right)$ & -2.497 \\
\hline
\end{tabular}

a) $R_{1}=\Sigma|| F_{\mathrm{o}}|-| F_{\mathrm{c}}|| \Sigma\left|F_{\mathrm{o}}\right|$; b) $w R_{2}=\Sigma\left[w\left(F_{\mathrm{o}}{ }^{2}-F_{\mathrm{c}}{ }^{2}\right)^{2}\right] / \Sigma\left[w\left(F_{\mathrm{o}}{ }^{2}\right)^{2}\right]^{1 / 2}$ 
$\rightarrow$ 三维 $(2 \mathrm{D} \rightarrow 3 \mathrm{D})$ 的互锁结构. 其不对称单元包括 $\left[\mathrm{Mo}_{8} \mathrm{Na}_{2} \mathrm{O}_{26}\left(\mu_{2}-\mathrm{OH}\right)_{2}\right]^{4-}$ 阴离子、 $\mathrm{Ni}^{\mathrm{II}}$ 阳离子、Btp 配体 分子和水分子(图 1). 由图 1 可知, $\left[\mathrm{Mo}_{8} \mathrm{Na}_{2} \mathrm{O}_{26}\left(\mu_{2-}\right.\right.$ $\left.\mathrm{OH})_{2}\right]^{4-}$ 阴离子含有 8 个端基氧 $(\mathrm{O} t) 、 14$ 个 $\mu_{2}-\mathrm{O} 、 4$ 个 $\mu_{3}-\mathrm{O}$ 和 2 个 $\mu_{6}-\mathrm{O}$, 显示出与 $\left[\mathrm{V}_{10} \mathrm{O}_{28}\right]^{6-}$ 相似的结构特征, 包括 8 个 $\left[\mathrm{MoO}_{6}\right]$ 八面体和 2 个 $\left[\mathrm{NaO}_{6}\right]$ 八面体, 形成 10 个八面体共边排列、两个 $\left[\mathrm{Mo}_{4} \mathrm{O}_{13}\right]$ 子单元交错堆积的 结构. Na(1)-O 1.714(7)-2.491(7) ̊, O-Na(1)-O 73.4(2)$165.2(3)^{\circ}$, 这些数据与文献报道的键长键角范围一 致 ${ }^{[19]}$. 两个 $\mathrm{Na}^{\mathrm{I}}$ 通过 4 个端氧和一个 $\mu_{5}-\mathrm{O}$ 与 $\left[\mathrm{Mo}_{8} \mathrm{O}_{26}\right]^{4-}$ 阴离子配位, 形成具有 $\left[\mathrm{V}_{10} \mathrm{O}_{28}\right]^{6-}$ 结构特征的新多酸 阴离子 $\left[\mathrm{Mo}_{8} \mathrm{Na}_{2} \mathrm{O}_{26}\left(\mu_{2}-\mathrm{OH}\right)_{2}\right]^{4-}, \mathrm{Na}^{\mathrm{I}}$ 的另一端通过 $\mu_{2}-\mathrm{OH}^{[29]}(\mathrm{O} 14)$ 与 $\mathrm{Ni1}$ 连接, 这种情况在基于 $\left[\mathrm{Mo}_{8} \mathrm{O}_{26}\right]^{4}$

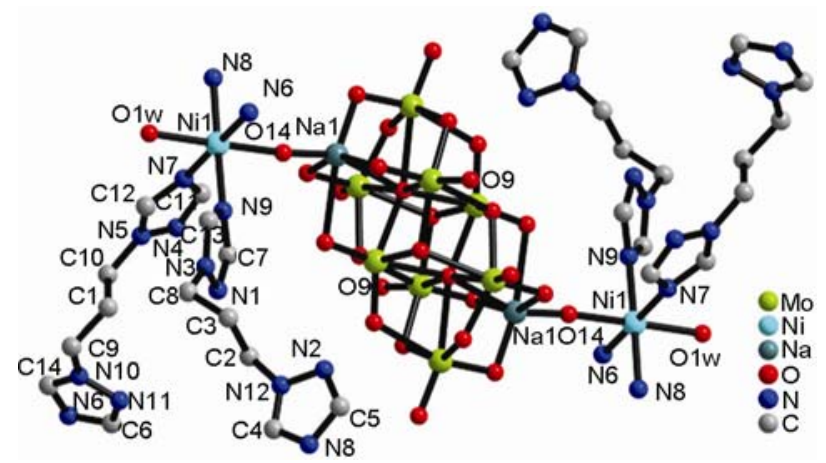

图 1 化合物 $\mathbf{1}\left[\mathrm{Ni}(\mathrm{Btp})_{2}\left(\mathrm{H}_{2} \mathrm{O}\right)\right]\left[\mathrm{Mo}_{8} \mathrm{Na}_{2} \mathrm{O}_{26}\left(\mu_{2}-\mathrm{OH}\right)_{2}\right]_{0.5} \cdot \mathrm{H}_{2} \mathrm{O}$ 的配位单元结构图(为了清晰, 游离水已经删去)

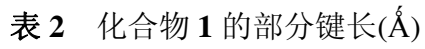

\begin{tabular}{cccc}
\hline $\mathrm{Ni}(1)-\mathrm{N}(6)$ & $2.057(6)$ & $\mathrm{Na}(1)-\mathrm{O}(6)$ & $1.847(8)$ \\
$\mathrm{Ni}(1)-\mathrm{N}(8)$ & $2.069(6)$ & $\mathrm{Na}(1)-\mathrm{O}(5)$ & $2.207(7)$ \\
$\mathrm{Ni}(1)-\mathrm{O}(14)$ & $2.072(5)$ & $\mathrm{Na}(1)-\mathrm{O}(10)$ & $2.330(6)$ \\
$\mathrm{Ni}(1)-\mathrm{N}(7)$ & $2.083(6)$ & $\mathrm{Na}(1)-\mathrm{O}(13)$ & $2.352(9)$ \\
$\mathrm{Ni}(1)-\mathrm{N}(9)$ & $2.116(6)$ & $\mathrm{Na}(1)-\mathrm{O}(8) \# 1$ & $2.491(7)$ \\
$\mathrm{Ni}(1)-\mathrm{O}(1 \mathrm{~W})$ & $2.126(5)$ & $\mathrm{Na}(1)-\mathrm{O}(14)$ & $1.714(7)$ \\
$\mathrm{Mo}(1)-\mathrm{O}(8)$ & $1.685(5)$ & $\mathrm{Mo}(3)-\mathrm{O}(10)$ & $2.330(5)$ \\
$\mathrm{Mo}(1)-\mathrm{O}(10) \# 1$ & $2.182(5)$ & $\mathrm{Mo}(1)-\mathrm{O}(4)$ & $1.721(5)$ \\
$\mathrm{Mo}(1)-\mathrm{O}(10)$ & $2.341(5)$ & $\mathrm{Mo}(1)-\mathrm{O}(12)$ & $1.948(5)$ \\
$\mathrm{Mo}(2)-\mathrm{O}(2)$ & $1.691(6)$ & $\mathrm{Mo}(1)-\mathrm{O}(11)$ & $1.953(5)$ \\
$\mathrm{Mo}(2)-\mathrm{O}(13)$ & $1.709(6)$ & $\mathrm{Mo}(4)-\mathrm{O}(1)$ & $1.713(6)$ \\
$\mathrm{Mo}(2)-\mathrm{O}(7)$ & $1.858(5)$ & $\mathrm{Mo}(4)-\mathrm{O}(6)$ & $1.735(6)$ \\
$\mathrm{Mo}(2)-\mathrm{O}(12) \# 1$ & $2.032(5)$ & $\mathrm{Mo}(4)-\mathrm{O}(3)$ & $1.946(6)$ \\
$\mathrm{Mo}(2)-\mathrm{O}(11)$ & $2.270(5)$ & $\mathrm{Mo}(4)-\mathrm{O}(7)$ & $1.968(6)$ \\
$\mathrm{Mo}(2)-\mathrm{O}(10)$ & $2.369(5)$ & $\mathrm{Mo}(4)-\mathrm{O}(4)$ & $2.333(5)$ \\
$\mathrm{Mo}(3)-\mathrm{O}(5)$ & $1.674(6)$ & $\mathrm{Mo}(4)-\mathrm{O}(10)$ & $2.400(5)$ \\
$\mathrm{Mo}(3)-\mathrm{O}(9)$ & $1.703(5)$ & $\mathrm{Mo}(3)-\mathrm{O}(12)$ & $2.316(5)$ \\
$\mathrm{Mo}(3)-\mathrm{O}(11) \# 1$ & $2.001(5)$ & $\mathrm{Mo}(3)-\mathrm{O}(3)$ & $1.915(6)$ \\
\hline
\end{tabular}

注: 用于产生等效原子的对称代码 $\# 1-x+2,-y,-z+1$
的金属-有机体系中尚属首例，是该化合物有趣特点之 一. 该化合物中每个晶体学独立的 $\mathrm{Ni}^{\mathrm{II}}$ 离子都是六配 位的，与来自 4 个 Btp 配体的 4 个 $\mathrm{N}$ 原子 $(\mathrm{N} 6, \mathrm{~N} 7, \mathrm{~N} 8$ 和 N9), 一个桥连 $\mathrm{Na} 1$ 的 $\mu_{2}-\mathrm{OH}(\mathrm{O} 14)$ 和一个来自水中 的氧原子 $(\mathrm{O} 1 \mathrm{w})$ 配位形成扭曲的八面体配位模式, $\mathrm{Ni}(1)-\mathrm{N} \quad 2.057(6) \sim 2.116(6) \AA ， \quad \mathrm{Ni}(1)-\mathrm{O} \quad$ 2.072(5) 2.126(5) $\AA, \quad \mathrm{N}-\mathrm{Ni}(1)-\mathrm{N} \quad 87.5(2) \sim 178.8(2)^{\circ}, \quad \mathrm{O}-\mathrm{Ni}(1)-\mathrm{O}$ $178.0(2)^{\circ}$. 每个晶体学独立的 $\mathrm{Na}^{\mathrm{I}}$ 离子同样是六配位 的, 分别与来自 $\left[\mathrm{Mo}_{8} \mathrm{Na}_{2} \mathrm{O}_{26}\left(\mu_{2}-\mathrm{OH}\right)_{2}\right]^{4-}$ 的 4 个 $\mathrm{O} t$ 、一 个 $\mu_{5}-\mathrm{O}$ 和一个桥连 Ni1 的 $\mu_{2}-\mathrm{OH}(\mathrm{O} 14)$ 配位, 采取扭 曲的八面体配位模式. 每一个 $\mathrm{Ni}^{\mathrm{II}}$ 离子通过 4 个 Btp 配体分子和相邻的 4 个 $\mathrm{Ni}^{\mathrm{II}}$ 离子连接形成一个沿 $a c$ 面 的二维格子层(图 2), 由于沿 $a$ 轴和 $c$ 轴配体的构型不 同，使两个方向的 $\mathrm{Ni} \cdots \mathrm{Ni}$ 距离不同，分别为 11.687 和 $12.428 \AA$. 这些格子层都是成对的且相互平行.

$\left[\mathrm{Mo}_{8} \mathrm{Na}_{2} \mathrm{O}_{26}\left(\mu_{2}-\mathrm{OH}\right)_{2}\right]^{4-}$ 阴离子作为建筑单元，通 过 $\mathrm{Na}^{\mathrm{I}}$ 离子的桥连, 将相邻的格子层连接成一个夹心 型的二维柱状层 (图 3(a)). 层与层之间的距离为 $14.084 \AA$ ，见图 3(b). 化合物 $\mathbf{1}$ 中一个最吸引人的特 点是每一个柱状层都与 $a c$ 面平行, 并且以一种交错 的方式与邻近的柱状层互锁(沿 $a$ 轴), 从而形成了一 个 $2 \mathrm{D} \rightarrow 3 \mathrm{D}$ 的互锁结构(图 4). 每一个柱状层结构中 多酸的端基氧和另一个柱状层结构中的配位水氧通 过氢键作用 $(\mathrm{O} 1 \mathrm{~W}-\mathrm{H} \cdots \mathrm{O} 9=2.8074 \AA)$ 进一步稳固整 个框架，形成了 $3 \mathrm{D}$ 的超分子结构. 这种基于多酸的 $2 \mathrm{D} \rightarrow 3 \mathrm{D}$ 互锁结构并不多见，化合物 1 是首例以交错 方式互锁的多酸基化合物，它不同于陈亚光等报道 的以平行方式互锁的结构 ${ }^{[26]}$.

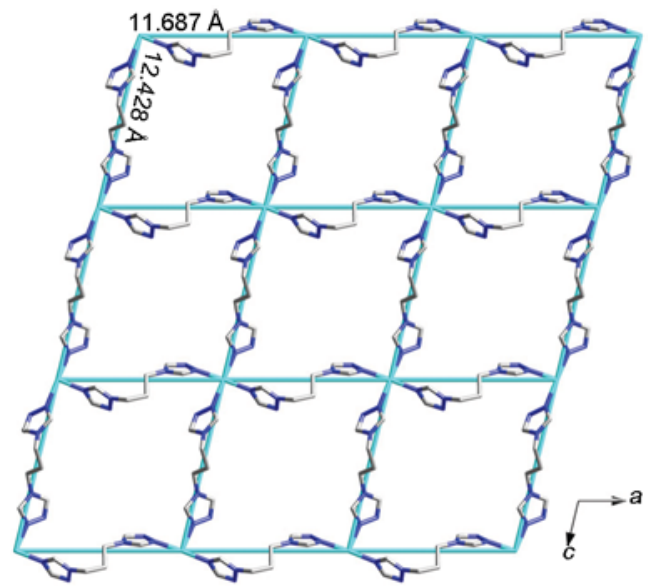

图 2 化合物 1 的二维格子结构 
(a)
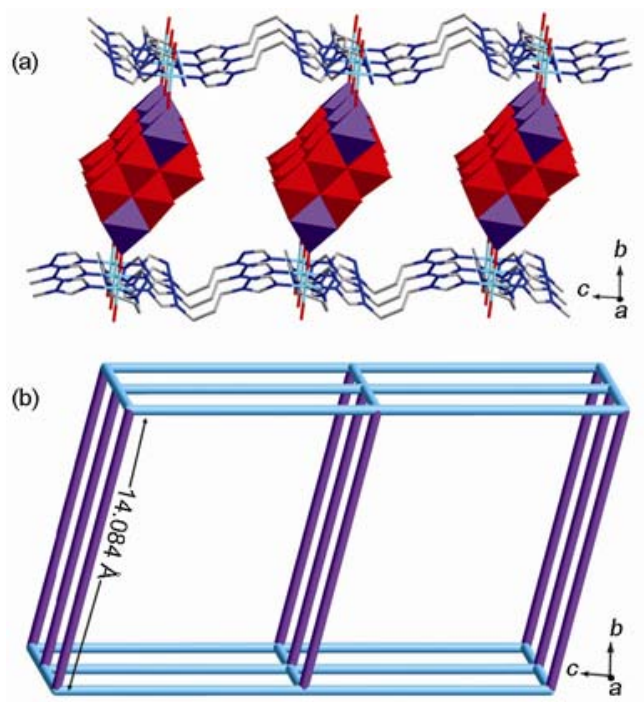

图 3 标题化合物的柱状层结构. (a)棍/多面体图, (b)拓扑结 构图

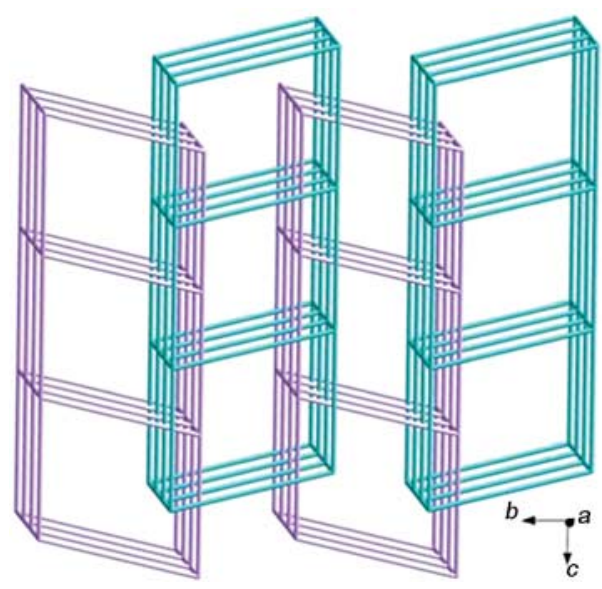

图 4 化合物 1 的交错互锁结构示意图

\section{2 化合物 1 的热分析}

热分析表明, 化合物 $\mathbf{1}$ 在室温到 $800{ }^{\circ} \mathrm{C}$ 范围内 有三步明显失重, 第一步在 $60 \sim 250{ }^{\circ} \mathrm{C}$ 之间, 质量损 失为 $3.68 \%$ (理论值 $3.33 \%$ ), 对应于失去 1 个结晶水分 子和 1 个结构水分子; 第二步失重在 320 620 ${ }^{\circ} \mathrm{C}$ 之 间, 质量损失为 $48.37 \%$ (理论值 $48.48 \%$ ), 对应于失去 2 个 Btp 配体分子. 前两步的总失重 $52.05 \%$ 与理论失 重值 $51.81 \%$ (2 个水分子和 2 个配体分子) 基本吻合. 当温度高于 $670{ }^{\circ} \mathrm{C}$ 后, 出现一个急剧的失重过程, 直到 $800{ }^{\circ} \mathrm{C}$ 也没有结束, 这可能是由于 $\mathrm{MoO}_{3}$ 的升华 所致 ${ }^{[30]}$.

\section{3 化合物 1 本体修饰碳糊电极的电化学行为}

化合物 1 本体修饰碳糊电极 $(\mathbf{1}-\mathrm{CPE})$ 的电化学研 究在 $1.0 \mathrm{~mol} / \mathrm{L} \mathrm{H}_{2} \mathrm{SO}_{4}$ 水溶液中进行. 在 $+400 \sim-300$ $\mathrm{mV}$ 的电位范围内, 1-CPE上有一对明显的氧化-还原 峰, 其平均峰电位为 $114 \mathrm{mV}\left[E_{1 / 2}=\left(E_{\mathrm{pa}}+E_{\mathrm{pc}}\right) / 2\right]$. 此 峰应归属于 $\mathrm{Mo}(\mathrm{VI}) / \mathrm{Mo}(\mathrm{V})$ 的氧化还原 ${ }^{[31 ~ 32]}$. 图 5 给 出了 1-CPE 在不同扫速下的循环伏安图. 当扫速由 $40 \mathrm{mV} / \mathrm{s}$ 逐渐增至 $450 \mathrm{mV} / \mathrm{s}$ 时, 峰电位逐渐发生变 化: 随扫速的增大, 阴极峰电位逐渐向负方向移动, 阳极峰电位逐渐向正方向移动. 峰电流随扫速的变 化情况见插图, 在测定的扫速范围内, 阴极峰和阳极 峰电流与扫速成直线关系, 表明这个氧化-还原过程 是表面控制过程.

图 6 给出了在 $1.0 \mathrm{~mol} / \mathrm{L} \mathrm{H}_{2} \mathrm{SO}_{4}$ 水溶液中亚硝酸 盐在 1-CPE 上的电催化还原图. 图中可见，随着亚硝

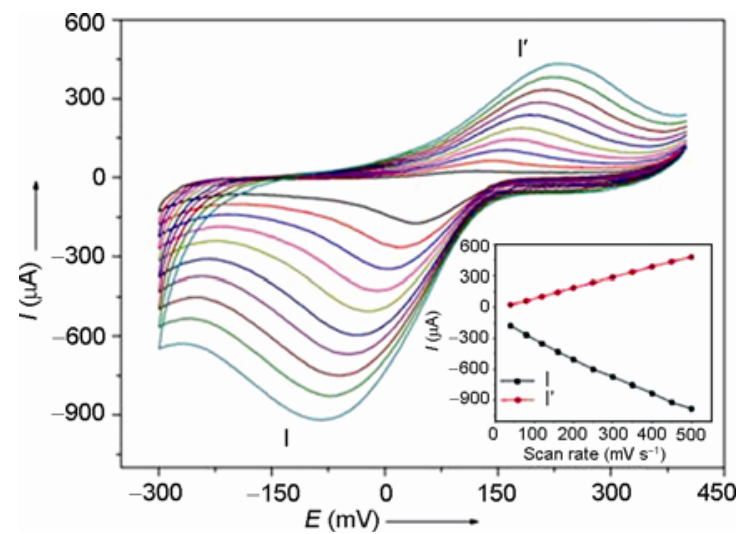

图 51 - $\mathrm{CPE}$ 在 $1.0 \mathrm{~mol} / \mathrm{L} \mathrm{H}_{2} \mathrm{SO}_{4}$ 水溶液中不同扫速下 (从里 到外: $40,80,120,160,200,250,300,350,400$ 和 $450 \mathrm{mV} / \mathrm{s}$ )的 循环伏安图 (插图: 峰电流与扫速的关系)

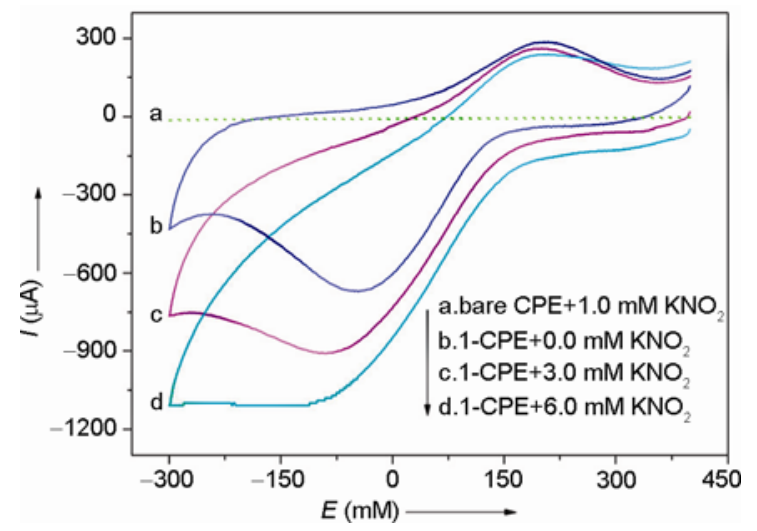

图 6 1-CPE 在含有不同浓度 $\mathrm{KNO}_{2}$ 的 $1.0 \mathrm{~mol} / \mathrm{L} \mathrm{H}_{2} \mathrm{SO}_{4}$ 水溶 液中的循环伏安图(扫速: $300 \mathrm{mV} / \mathrm{s}$ ) 
酸钾浓度的增大, 还原峰电流逐渐增大, 而相应的氧 化峰电流逐渐降低, 这表明化合物 $\mathbf{1}$ 中 $\left[\mathrm{Mo}_{8} \mathrm{Na}_{2} \mathrm{O}_{26}\left(\mu_{2}-\right.\right.$ $\left.\mathrm{OH})_{2}\right]^{4}$ 阴离子对亚硝酸根的还原具有电催化作用 ${ }^{[33,34]}$.

\section{4 化合物 1 的光催化性质}

亚甲基蓝(MB)作为一种典型的染料，可以用来 评估光催化剂的催化活性 ${ }^{[35,36]}$. 我们研究了化合物 $\mathbf{1}$ 在紫外灯照射下对 $\mathrm{MB}$ 的光催化降解作用. 实验中, 将 $90 \mathrm{~mL} 10.0 \mathrm{mg} / \mathrm{L}$ 亚甲基蓝和 $50 \mathrm{mg}$ 化合物 $\mathbf{1}$ 加入 烧杯中, 在超声条件下分散 $0.5 \mathrm{~h}$, 构成悬浮体系. 在 $8 \mathrm{~W}$ 的录灯照射下进行光催化降解实验, 并不停的摚 拌. 每隔 $20 \mathrm{~min}$ 取一组样品, 测试其吸收曲线 (图 7). 在开始的 $20 \mathrm{~min}$ 内, 大约 $50 \%$ 的 MB 已降解, 降 解速率为 $1.534 \mathrm{mg} / \mathrm{L} / \mathrm{h} .160 \mathrm{~min}$ 后, 接近 $80 \%$ 的 $\mathrm{MB}$ 降解完. 作为对照, 在相同的条件下进行了不加化合

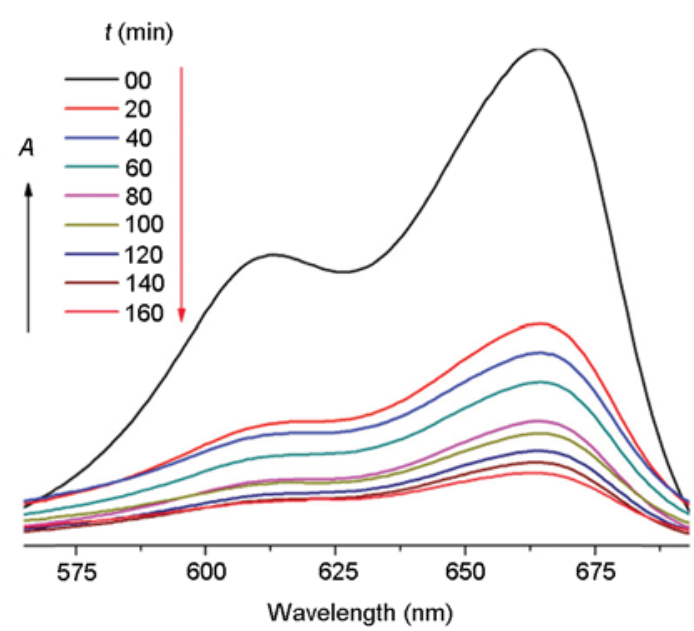

图 7 在紫外灯照射下, 化合物 $\mathbf{1}$ 对 $\mathrm{MB}$ 溶液光催化降解反 应过程的吸收光谱 $\left(\lambda_{\max }=664 \mathrm{~nm}\right)$
物 1 的光催化降解 MB 实验, $160 \mathrm{~min}$ 后, MB 降解不 足 10\%, 表明化合物 $\mathbf{1}$ 对 MB 的降解有较高的光催化 活性.

\section{5 化合物 1 的苂光性质}

室温下, 分别测定了 Btp 配体和化合物 $\mathbf{1}$ 的固体 荧光光谱. 自由配体的激发光谱在 $310 \mathrm{~nm}$ 附近有吸 收, 用该波长的光进行激发, 在 $418 \mathrm{~nm}$ 附近出现一 个宽的配体荧光发射峰，对应于配体内 $\pi * \rightarrow \mathrm{n}$ 跃 迁 ${ }^{[37]}$. 化合物 1 的激发波长在 $350 \mathrm{~nm}$ 附近有吸收, 其发射光谱显示一个主峰在 $470 \mathrm{~nm}$ 处, 并且在 454 $\mathrm{nm}$ 处存在一个肩峰. 化合物 $\mathbf{1}$ 的这个肩峰很可能是 因为配体间的能量传递造成的 ${ }^{[38]}$. 所以化合物 $\mathbf{1}$ 的苂 光行为主要归属于配体到金属的电荷传递 $(\mathrm{LMCT})^{[39]}$.

\section{4 结论}

利用水热法合成了一个结构新颖的基于新型混 金属的多酸簇阴离子 $\left[\mathrm{Mo}_{8} \mathrm{Na}_{2} \mathrm{O}_{26}\left(\mu_{2}-\mathrm{OH}\right)_{2}\right]^{4-}$ 和 $\mathrm{Ni}$ - 有 机双三唑配体的 $2 \mathrm{D}$ 配位聚合物 $\left[\mathrm{Ni}(\mathrm{Btp})_{2}\left(\mathrm{H}_{2} \mathrm{O}\right)\right]$ $\left[\mathrm{Mo}_{8} \mathrm{Na}_{2} \mathrm{O}_{26}\left(\mu_{2}-\mathrm{OH}\right)_{2}\right]_{0.5} \cdot \mathrm{H}_{2} \mathrm{O}$. 该化合物通过 $\left[\mathrm{Mo}_{8} \mathrm{Na}_{2}-\right.$ $\left.\mathrm{O}_{26}\left(\mu_{2}-\mathrm{OH}\right)_{2}\right]^{4-}$ 中的 $\mathrm{Na}^{\mathrm{I}}$ 连接相邻的金属 $\mathrm{Ni}$-有机配体 二维格子层, 形成一种夹心型柱状层. 相邻的柱状层 以一种交错的方式互锁，从而形成了一个特殊的 2D $\rightarrow 3 \mathrm{D}$ 的互锁结构. 标题化合物表现出了较好的电化 学、荧光以及光催化性质, 其成功合成不仅证实了多 酸结构的多样性，丰富了多酸基杂化化合物，而且为 光催化降解环境污染物提供了一种新催化剂，相关 化合物合成与性能的深入研究还在进行中.

\section{参考文献}

1 Liu T, Diemann E, Li H, Dress AWM, Müller A. Self-assembly in aqueous solution of wheel-shaped $\mathrm{Mo}_{154}$ oxide clusters into vesicles. Nature, 2003, 426: 59-62

2 Zeng H, Newkome GR, Hill CL. Poly(polyoxometalate) dendrimers: molecular prototypes of new catalytic materials. Angew Chem Int Ed, 2000, 39: 1771-1774

3 王恩波, 胡长文, 许林. 多酸化学导论. 北京: 化学工业出版社, 1998. 1-38

4 Coronado E, Gmóez-García CJ. Polyoxometalate-based molecular materials. Chem Rev, 1998, 98: 273-296 
5 Müller A, Kögerler P. Erratum to "from simple building blocks to structures with increasing size and complexity". Coord Chem Rev, 2000, 199: 335-341

6 Jiang CJ, Lesbani A, Kawamoto R, Uchida S, Mizuno N. Channel-selective independent sorption and collection of hydrophilic and hydrophobic molecules by $\mathrm{Cs}_{2}\left[\mathrm{Cr}_{3} \mathrm{O}\left(\mathrm{OOCC}_{2} \mathrm{H}_{5}\right)_{6}\left(\mathrm{H}_{2} \mathrm{O}\right)_{3}\right]_{2}\left[\alpha-\mathrm{SiW}_{12} \mathrm{O}_{40}\right]$ ionic crystal. J Am Chem Soc, 2006, 128: 14240-14241

7 Hagrman D, Zubieta C, Rose DJ, Zubieta J, Haushalter RC. Composite solids constructed from one-dimensional coordination polymer matrices and molybdenum oxide subunits: polyoxomolybdate clusters within [ $\left.\left\{\mathrm{Cu}\left(4,4^{\prime}-\text { bpy }\right)\right\}_{4} \mathrm{Mo}_{8} \mathrm{O}_{26}\right]$ and $\left[\left\{\mathrm{Ni}_{(}\left(\mathrm{H}_{2} \mathrm{O}\right)_{2}\left(4,4^{\prime}-\text {-bpy }\right)_{2}\right\}_{2} \mathrm{Mo}_{8} \mathrm{O}_{26}\right]$ and one-dimensional oxide chains in $\left[\left\{\mathrm{Cu}\left(4,4^{\prime} \text {-bpy }\right)\right\}_{4} \mathrm{Mo}_{15} \mathrm{O}_{47}\right] \cdot 8 \mathrm{H}_{2} \mathrm{O}$. Angew Chem Int Ed, 1997, 36: 873-876

8 Tian AX, Ying J, Peng J, Sha JQ, Han ZG, Ma JF, Su ZM, Hu NH, Jia HQ. Assembly of the highest connectivity Wells-Dawson polyoxometalate coordination polymer: the use of organic ligand flexibility. Inorg Chem, 2008, 47: 3274-3283

9 Reinoso S, Vitoria P, Felices LS, Montero A, Lezama L, Gutiérrez-Zorrilla JM. Tetrahydroxy-p-benzoquinone as a source of polydentate O-donor ligands. Synthesis, crystal structure, and magnetic properties of the $[\mathrm{Cu}(\mathrm{bpy})(\mathrm{dhmal})]_{2}$ dimer and the two-dimensional $\left[\left\{\mathrm{SiW}_{12} \mathrm{O}_{40}\right\}\left\{\mathrm{Cu}_{2}(\text { bpy })_{2}\left(\mathrm{H}_{2} \mathrm{O}\right)(\mathrm{ox})\right\}_{2}\right] \cdot 16 \mathrm{H}_{2} \mathrm{O}$ inorganic-metalorganic hybrid. Inorg Chem, 2007, 46: 1237-1249

10 Lin HS, Maggard PA. Synthesis and structures of a new series of silver-Vanadate hybrid solids and their optical and photocatalytic properties. Inorg Chem, 2008, 47: 8044-8052

11 王敬平, 马鹏涛, 牛景杨. 新型有机-无机杂化的类 Dawson 型多金属钨酸盐 $\left[\mathrm{Co}\left(2,2^{\prime}-\mathrm{bpy}\right)_{3}\right]_{2}\left[\mathrm{Co}\left(2,2^{\prime}-\mathrm{bpy}\right)_{2} \mathrm{Cl}\right]\left[\mathrm{Co}\left(2,2^{\prime}-\mathrm{bpy}\right)_{2}\right]$ $\mathrm{H}_{2}\left[\mathrm{SbW}_{18} \mathrm{O}_{60}\right] \cdot 4 \mathrm{H}_{2} \mathrm{O}$ 的合成、晶体结构及性质. 中国科学 B 辑: 化学, 2007, 37: 575-580

12 Hagrmann D, Zubieta C, Haushalter RC, Zubieta J. Composite solids constructed from one-dimensional coordination polymer matrices and molybdenum oxide subunits: Polyoxomolybdate clusters within $\left[\left\{\mathrm{Cu}\left(4,4^{\prime}-\text { bpy }\right)\right\}_{4} \mathrm{Mo}_{8} \mathrm{O}_{26}\right]$ and $\left[\left\{\mathrm{Ni}\left(\mathrm{H}_{2} \mathrm{O}\right)_{2}\left(4,4^{\prime}-\text {-bpy }\right)_{2}\right\}_{2} \mathrm{Mo}_{8} \mathrm{O}_{26}\right]$ and one-dimensional oxide chains in $\left[\left\{\mathrm{Cu}\left(4,4^{\prime} \text {-bpy }\right)\right\}_{4} \mathrm{Mo}_{15} \mathrm{O}_{47}\right] \cdot 8 \mathrm{H}_{2} \mathrm{O}$. Angew Chem Int Ed, 1997, 36: 873-876

13 Bridgeman AJ. The electronic structure and stability of the isomers of octamolybdate. J Phys Chem A, 2002, 106: 12151-12160

$14 \mathrm{Wu} \mathrm{CD}, \mathrm{Lu} \mathrm{CZ}$, Zhuang HH, Huang JS. Hybrid coordination polymer constructed from $\beta$-octamolybdates linked by quinoxaline and its oxidized product benzimidazole coordinated to binuclear copper(I) fragments. Inorg Chem, 2002, 41: 5636-5637

15 Hageman D, Zapf PJ, Zubieta J. A two-dimensional network constructed from hexamolybdate, octamolybdate and $\left[\mathrm{Cu}_{3}\left(4,7-\mathrm{phen}_{3}\right]^{3+}\right.$ clusters: $\left[\left\{\mathrm{Cu}_{3}(4,7-\mathrm{phen})_{3}\right\}_{2}\left\{\mathrm{Mo}_{14} \mathrm{O}_{45}\right\}\right]$. Chem Commun, 1998, 12: 1283-1284

16 Raring RS, Zubieta J. Hydrothermal synthesis and structural characterization of an organic-inorganic hybrid material: $\left(\mathrm{H}_{2} \text { tptz) }\right)_{2}\left[\delta-\mathrm{Mo}_{8} \mathrm{O}_{26}\right] \cdot 2 \mathrm{H}_{2} \mathrm{O}$ (tptz=2,4,6-tripyridyltriazine). Inorg Chim Acta, 2001, 312: 188-196

17 Zhai QG, Wu XY, Chen ZG, Lu CZ. Construction of Ag/1,2,4-triazole/polyoxometalates hybrid family varying from diverse supramolecular assemblies to 3-D rod-packing framework. Inorg Chem, 2007, 46: 5046-5058

18 Dong BX, Xu Q. Investigation of flexible organic ligands in the molybdate system: Delicate influence of a peripheral cluster environment on the isopolymolybdate. Inorg Chem, 2009, 48: 5861-5873

19 Honma N, Kutsuhiro K, Ozeki T. Self-assembly of a lacunary $\alpha$-Keggin undecatungstophosphate into a three-dimensional network linked by s-block cations. Chem Commun, 2002, 23: 2896-2897

20 An HY, Xu TQ, Zheng H, Han ZB. A new double-Keggin-anion-templated organic-inorganic hybrid architecture: Synthesis, crystal structure and photoluminescence. Inorg Chem Commun, 2010, 13: 302-305

21 Wang SM, Chen WL, Wang EB, Li YG, Feng XJ, Liu L. Three new polyoxometalate-based hybrids prepared from choline chloride/urea deep eutectic mixture at room temperature. Inorg Chem Commun, 2010, 13: 972-975

22 Wang XL, Qin C, Wang EB, Su ZM. An unusual polyoxometalate-encapsulating 3D polyrolytaxane framework formed by molecular squares threading on a twofold interpenetrated diamondoid skeleton. Chem Commun, 2007, 41: 4245-4247

23 Wang XL, Qin C, Wang EB, Su ZM, Li YG, Xu L. Self-assembly of nanometer-scale $\left[\mathrm{Cu}_{24} \mathrm{I}_{10} \mathrm{~L}_{12}\right]^{14+}$ cages and ball-shaped Keggin clusters into a (4,12)-connected 3D framework with photoluminescent and electrochemical properties. Angew Chem Int Ed, 2006, 45: 7411-7414

24 Zheng ST, Yang GY. The first polyoxometalate-templated four-fold interpenetrated coordination polymer with new topology and ferroelectricity. Dalton Trans, 2010, 39: 700-703

25 Kuang XF, Wu XY, Yu RM, Donahue JP, Huang JS, Lu CZ. Assembly of a metal-organic framework by sextuple intercatenation of discrete adamantane-like cages. Nature Chem, 2010, 2: 461-465

26 Zhang CJ, Pang HJ, Tang Q, Wang HY, Chen YG. Tailoring microstructures of isopolymolybdates: regular tuning of the ligand spacer length and metal coordination preferences . Dalton Trans, 2010, 39: 7993-7999

27 Liu XG, Zhang YM, Li BL. Synthesis and crystal structure of 1,4-bis(1,2,4-triazol-1-yl)butane. J Suzhou Univ (Natural Sci), 2005, 21: 59-62

28 Sheldrick GM. A short history of SHELX. Acta Crystallogr Sect. A Found. Crystallogr, 2008, 64: 112-12 
29 Yu F, Kong XJ, Zheng YY, Ren YP, Long LS, Huang RB, Zheng LS. pH-Dependent assembly of OD to 3D Keggin-based coordination polymers: Structures and catalytic properties. Dalton Trans, 2009, 43: 9503-9509

30 Wang XL, Bi YF, Chen BK, Lin HY, Liu GC. Self-assembly of organic-inorganic hybrid materials constructed from eight-connected coordination polymer hosts with nanotube channels and polyoxometalate guests as templates. Inorg Chem, 2008, 47: 2442-2448

31 Li YW, Wang YH, Li YG, Wang EB. A new supramolecular compound based on $\mathrm{Mn}^{\mathrm{III}}$-Schiff-base and $\beta$-octamolybdate. Inorg Chem Commun, 2009, 12: 112-115

32 Qin C, Wang XL, Qi YF, Wang EB, Hu CW, Xu L. A novel two-dimensional $\beta$-octamolybdate supported alkaline-earth metal complex: $\left[\mathrm{Ba}(\mathrm{DMF})_{2}\left(\mathrm{H}_{2} \mathrm{O}\right)\right]_{2}\left[\mathrm{Mo}_{8} \mathrm{O}_{26}\right] \cdot 2 \mathrm{DMF}$. J Solid State Chem, 2004, 177: 3263-3269

33 Sadakane M, Steckhan E. Electrochemical properties of polyoxometalates as electrocatalysts. Chem Rev, 1998, 98: 219-238

34 Chen SM. Bicatalyst electrocatalytic reduction and oxidation of nitrite by $\mathrm{Fe}(\mathrm{II})$ and $\mathrm{Cu}(\mathrm{II})$ complexes in the same solution. $J$ Electroanal Chem, 1998, 457: 23-30

$35 \mathrm{Hu}$ Y, Luo F, Dong FF. Design synthesis and photocatalytic activity of a novel lilac-like silver-vanadate hybrid solid based on dicyclic rings of $\left[\mathrm{V}_{4} \mathrm{O}_{12}\right]^{4-}$ with $\left\{\mathrm{Ag}_{7}\right\}^{7+}$ cluster. Chem Commun, 2010, DOI: 10.1039/c0cc02965c.

36 刘东, 曾祥武, 罗晓刚. 纳米四氧化三铁降解亚甲基蓝的研究. 化学与生物工程, 2010, 27: 28-30

37 Yang EC, Li J, Ding B, Liang QQ, Wang XG, Zhao XJ. An eight-connected 3D lead(II) metal-organic framework with octanuclear lead(II) as a secondary building unit: synthesis, characterization and luminescent property. CrystEngComm, 2008, 10: 158-161

38 Ashiry KO, Zhao YH, Shao KZ, Su ZM. Syntheses and characterizations of three coordination polymers based on dipyridylbenzoates and 1,4-bezenedicarboxylate. Polyhedron, 2009, 28: 975-979

39 Liu XG, Wang LY, Xia Z, Li BL, Zhang Y. Structural versatility of eight zinc(II) coordination polymers constructed with a long flexible ligand 1,4-bis(1,2,4-triazol-1-yl)-butane. Cryst Growth Des, 2009, 9: 3997-4005

\title{
A novel $2 \mathrm{D} \rightarrow 3 \mathrm{D}$ polycatenated coordination polymer based on a new $\left[\mathrm{Mo}_{8} \mathrm{Na}_{2} \mathrm{O}_{28}\right]^{6-}$ building unit: Assembly, structure and properties
}

\author{
WANG XiuLi, LI Jin, TIAN AiXiang, LIU GuoCheng \& LIN HongYan \\ Faculty of Chemistry and Chemical Engineering, Bohai University, Jinzhou 121000, China
}

\begin{abstract}
A novel 2D inorganic-organic hybrid coordination polymer based on polyoxometalate $\left[\mathrm{Mo}_{8} \mathrm{Na}_{2} \mathrm{O}_{28}\right]^{6-}$, $\left[\mathrm{Ni}(\mathrm{Btp})_{2}\left(\mathrm{H}_{2} \mathrm{O}\right)\right]\left[\mathrm{Mo}_{8} \mathrm{Na}_{2} \mathrm{O}_{26}\left(\mu_{2}-\mathrm{OH}\right)_{2}\right]_{0.5} \cdot \mathrm{H}_{2} \mathrm{O}(\mathbf{1})(\mathrm{Btp}=1,3$-bis(1,2,4-triazol-1-yl)propane), has been prepared under hydrothermal reaction with a mixture of $\left(\mathrm{NH}_{4}\right)_{6} \mathrm{Mo}_{7} \mathrm{O}_{24} \cdot 4 \mathrm{H}_{2} \mathrm{O}, \mathrm{NiCl}_{2} \cdot 6 \mathrm{H}_{2} \mathrm{O}$, flexible ligand (Btp), $\mathrm{NaOH}$ and water, and structurally characterized by elemental analyses, IR spectroscopy, TG, and single-crystal X-ray diffraction analysis. X-ray crystal structure analysis shows that, the compound belongs to monoclinic crystal system, space group $P 2{ }_{1} / c$, crystal data: $a=11.6871(8) \AA, b=21.7506(15) \AA, c=14.9208(8) \AA, Z=4, R_{1}=0.0487, w R_{2}=$ 0.1409 . Compound 1 contains a kind of new polyoxometalate cluster $\left[\mathrm{Mo}_{8} \mathrm{Na}_{2} \mathrm{O}_{28}\right]^{6-}$, which could be regarded as the transformation from $\beta$ - $\left[\mathrm{Mo}_{8} \mathrm{O}_{26}\right]^{4-}$ and with the structural characteristics similar to $\left[\mathrm{V}_{10} \mathrm{O}_{28}\right]^{6-}$ polyanion. The polyoxometalates are sandwiched by the $2 \mathrm{D}$ layer constructed by $\mathrm{Ni}^{\mathrm{II}}$ and $\mathrm{Btp}$, leading to the formation of sandwich-like 2D columnar layer. The 2D columnar layer penetrates each other and finally results in the construction of an interesting $2 \mathrm{D} \rightarrow 3 \mathrm{D}$ polycatenated structure. In addition, the title complex shows good electrocatalytic and photocatalytic activity.
\end{abstract}

Keywords: polyoxometalates, 2D polycatenated structure, coordination polymer, electrochemistry and fluorescence properties, photocatalytic activity 